\title{
Nutritional advances in patients with respiratory diseases
}

\author{
Annemie M.W.J. Schols
}

Affiliation: Dept of Respiratory Medicine, Maastricht University Medical Centre, NUTRIM School of Nutrition and Translational Research in Metabolism, Maastricht, The Netherlands.

Correspondence: Annemie M.W.J. Schols, Dept of Respiratory Medicine, Maastricht University Medical Centre, NUTRIM School of Nutrition and Translational Research in Metabolism, P.0. Box 5800, 6202 AZ Maastricht, The Netherlands. E-mail: a.scholsamaastrichtuniversity.nl

ABSTRACT Nutritional issues are increasingly recognised in the course of respiratory diseases, from primary prevention to advanced disease care. During the 2014 ERS International Congress in Munich, Germany, the topic was addressed in a Clinical Year in Review session. This review highlights new insights in generic and disease-specific nutritional issues from recently published peer-reviewed articles. The clinical relevance of obesity was highlighted in primary and secondary prevention across all respiratory diseases. Radiographic imaging was explored for characterisation of metabolic phenotypes as an integrated part of diagnostic work-up. Muscle regenerative defects and the autophagy-lysosome pathway were identified as new leads for combatting muscle wasting.

@ERSpublications

Nutritional issues need to be considered in respiratory disease, from primary prevention to advanced disease care http://ow.ly/GVI5D

\section{Introduction}

Nutrition and metabolism have been the topic of increasing scientific research in respiratory diseases. This is not surprising in the context of a paradigm shift in disease management from diagnosis and treatment to prediction and prevention. Next to smoking and physical activity, diet is an important and interrelated lifestyle factor not only for disease prevention but also contributing to heterogeneity in disease progression and prognosis. Traditionally, the nutritional interest in chronic obstructive pulmonary disease (COPD), cystic fibrosis (CF) and lung cancer has been on management of weight loss and muscle wasting (cachexia) in advanced disease stages, while obesity was predominantly investigated in relation to onset and progression of asthma and obstructive sleep apnoea syndrome. Obesity has now been identified as a risk factor across all respiratory diseases. Furthermore, combined with ageing-induced changes in muscle mass (sarcopenia), the clinical significance of sarcopenic obesity has been highlighted in COPD [1] and lung cancer [2].

\section{Adiposity and respiratory disease risk}

Body mass index (BMI) is commonly used to define obesity. Using a Mendelian randomisation approach in the Avon longitudinal study of parents and children, Granell et al. [3] provided evidence for a causal effect of high BMI on asthma risk. However, BMI does not consider inter-individual variations in proportion and distribution of body fat. In a cross-sectional study of 10981 school children, YIALLOUROS et al. [4] showed that in contrast to the linear association observed with BMI, body fat percentage displayed a U-shaped association with asthma. CHEN et al. [5] showed that central obesity most accurately predicted asthma in the nationwide Taiwan Children Health Study. HaN et al. [6] reported that adiposity indicators were associated with asthma in children with exhaled nitric oxide fraction ( $F$ eNO) as a

Received: Nov 152014 | Accepted after revision: Dec 202014

Conflict of interest: None declared.

Provenance: Submitted article, peer reviewed.

Copyright OERS 2015. ERR articles are open access and distributed under the terms of the Creative Commons Attribution Non-Commercial Licence 4.0. 
biomarker of eosinophilic airway inflammation and that among children with asthma, adiposity was associated with worse asthma severity or control in those with high FeNO. In 2014, several studies also reported an increased prevalence of overweight and obesity in patients with CF even in those with pancreatic insufficiency [7-10]. Generally, however, overweight CF patients had milder genotypes and better lung function. The clinical significance of obesity (management) in CF needs to be determined. Using data on anthropometric measurements and physical activity from 113279 participants in the National Institutes of Health-AARP Diet and Health Study, including 3648 new COPD diagnoses, BEHRENs et al. [11] showed that the incidence of COPD was higher in both severely obese (BMI $>35 \mathrm{~kg} \cdot \mathrm{m}^{-2}$ ) and underweight (BMI $<18.5 \mathrm{~kg} \cdot \mathrm{m}^{-2}$ ) participants, but after adjustment for waist circumference this only remained significant for underweight subjects. A larger waist circumference and higher waist-hip ratio were also positively associated with COPD.

\section{Understanding COPD complexity from a metabolic perspective}

Overweight and obesity generally contribute to increased cardiovascular disease risk while involuntary loss of body weight and muscle wasting affects physical functioning. It is well established in COPD that both musculoskeletal impairment and elevated cardiovascular risk are common, contributing to increased morbidity and mortality. It is not yet clear whether COPD is somehow influencing the development of these comorbidities or whether one should consider them (including COPD) as multimorbidity resulting from the interaction between genetic predisposition and overlapping lifestyle determinants. In addition to smoking and physical inactivity, evidence is accumulating that dietary habits are not only involved in the development of metabolic and cardiovascular diseases but may also influence aetiology and progression of COPD [12-14].

Using an unbiased statistical approach, VANFLETEREN et al. [15] identified five clusters of patients based on 13 objectively identified comorbidities in a cohort of 213 patients with moderate-to-severe COPD, attending a pulmonary rehabilitation programme. The five clusters were "less comorbidity", "cardiovascular", "cachectic", "metabolic" and "psychological". These clusters were comparable in terms of airflow limitation severity. A selection of systemic inflammatory markers were investigated and were generally comparable between the clusters except for a higher IL-6 level in the cardiovascular cluster and higher levels of soluble tumour necrosis factor receptors in the metabolic cluster; both clusters were also characterised by an elevated Framingham 10-year cardiovascular risk score. The cachectic cluster was characterised by lower diffusion capacity and more hyperinflation, in line with hypothesis driven approaches explaining why the emphysematous phenotype in particular is prone to weight loss and cachexia as recently reviewed by ScHOLs et al. [16].

VAN REMOORTEL et al. [17] investigated the effects of smoking and ageing on the prevalence of premorbid risk factors and comorbid conditions as well as their association with objectively assessed daily physical activity in the preclinical stages of COPD. They studied a population-based sample of 560 subjects with a new diagnosis of mild-to-moderate COPD by spirometry screening. The results were compared with those of 60 smoking controls with normal lung function and 60 never-smoker subjects, all of them age matched. The main results showed that premorbid risk factors and comorbid diseases were significantly higher in preclinical COPD than in never-smokers but were similar to smoking controls. Physical inactivity and smoking were identified as independent risk factors for the presence of two or more comorbidities. Overall, these observations challenge the concept that, for some comorbidities, COPD is an independent risk factor by showing that physical inactivity and smoking, but not COPD as such, are associated with their development.

To investigate whether age-related decline of lean mass and physical function is accelerated by obstructive lung disease or smoking, VAN DEN BORST et al. [18] investigated baseline data and 7-year longitudinal data from 260 patients with obstructive lung disease, 157 smoking controls, 866 former smoking controls and 891 never-smoker controls participating in the Health, Ageing and Body Composition (Health ABC) study. At baseline the mean age was 74 years [3]. In this initially well-functioning cohort it was shown that older adults with mild-to-moderate obstructive lung disease and smokers without obstructive lung disease had a comparable compromised baseline profile of body composition and physical functioning, while 7-year longitudinal trajectories were, to a large extent, comparable to those observed in never-smokers without obstructive lung disease. This suggests a common insult earlier in life related to smoking affects musculoskeletal function. Within the same Health ABC cohort, vAN DEN BORST et al. [19] also studied COPD-specific effects on visceral fat mass (assessed by computed tomography (CT)). Propensity scores were used to match 729 persons with normal lung function to 243 persons with obstructive pulmonary disease. Matching was based on age, sex, race, clinic site, BMI and smoking status. This study demonstrated a presence of excessive abdominal visceral fat in obstructive lung disease patients despite a similar total and subcutaneous fat mass, contributing to increased plasma IL-6 which in turn was strongly associated with all-cause and cause-specific mortality after 9.4 years follow-up. It is of interest to note that obstructive lung disease patients, despite a similar caloric content of the diet, were characterised by a 
significantly lower dietary quality as reflected by a higher intake of saturated fat, cholesterol and trans fat, as well as a lower intake of total dietary fibres and vitamin C. This coincided with a lower physical activity level and further illustrates the need to consider lifestyle factors integrative in combatting elevated cardiovascular risk in this population.

Next to abdominal obesity, a decreased skeletal muscle oxidative capacity in COPD due to loss of muscle mass and a muscle fibre type I to > II shift may also be a driver of elevated metabolic and cardiovascular risk [20]. "Metabolic flexibility" is defined as the ability to modify fuel oxidation in response to changes in nutrient availability. Low muscle oxidative capacity reduces the capacity to oxidise fatty acids and during fat overload (e.g. when overeating) may lead to accumulation of intramyocellular lipids and oxidative stress. A decreased muscle oxidative phenotype and mitochondrial dysfunction has been well established in advanced COPD but was also recently reported in less advanced airflow obstruction (mean forced expiratory volume in $1 \mathrm{~s}$ (FEV1) 58\%) [21]. In mild COPD (mean FEV1 96\%), GAGNon et al. [22] showed a preserved muscle oxidative phenotype but a reduced potential for angiogenesis. However, patients were characterised by reduced levels of physical activities and evidence of systemic inflammation suggesting that these factors precede the development of overt limb muscle dysfunction in COPD. Translational research by Remels et al. [23], using COPD as a clinical model, showed that inflammation affects skeletal muscle oxidative phenotype through activation of classical nuclear factor- $\kappa \mathrm{B}$ and reduction of IкB kinase $\alpha$ expression. In a large cohort of COPD patients, NATANEK et al. [24] showed great heterogeneity in muscle morphology and function overlapping those in sedentary healthy subjects. Using an unsupervised clustering method and post hoc validation, Gouzi et al. [25] tested whether muscle heterogeneity is an attribute of different phenotypes of the disease. They identified and validated two phenotypes of COPD patients showing a different peripheral muscle histomorphology and level of oxidative stress. Cluster 1 "atrophic" COPD patients showed reduced BMI, fat-free mass index, fibre cross-sectional area and increased oxidative stress, while cluster 2 COPD patients showed a moderate fibre type switch compared to sedentary controls. Unfortunately, the study did not include a measure of emphysema but was in line with the "cachectic" phenotype proposed by VANFLETEREN et al. [15]. The degree of hyperinflation assessed by residual volume was higher in the "atrophic" cluster and the degree of airflow obstruction was less in cluster 2 (mean FEV1 52\% versus 30\%), but was in line with the study population of VAN DEN BORST et al. [21]. To unravel if low muscle mass resembles a phenotypic characteristic of the emphysematous phenotype or whether emphysema per se (also) induces cachexia, longitudinal studies are needed including measures of respiratory pathology, muscle mass and ideally also molecular signatures in muscle biopsies that can be compared with cachexia induced by other diseases.

The observation of elevated visceral adipose tissue points towards an increased risk for ectopic fat in patients with COPD. Using CT, MADDOCKS et al. [26] reported an increased proportion of intramuscular fat in 101 patients with COPD compared to 10 healthy controls. Within the patient population associations with physical activity, exercise capacity and fibre type shift were shown. The combined studies indicate that altered peripheral skeletal muscle mass and metabolism combined with ectopic fat deposition may not only affect physical performance but also contribute to increased cardiovascular disease risk in COPD even in those without obesity. In the Korean National Health and Nutrition Examination Survey from 2008 to 2011, Chung et al. [1] reported an association between sarcopenia with abdominal obesity and metabolic syndrome in COPD.

\section{New developments in body composition assessment to characterise metabolic phenotypes}

In order to identify the different metabolic phenotypes and related risk in respiratory disease, an ERS Task Force on nutrition in COPD proposed to include involuntary weight loss and body composition assessment in addition to BMI in diagnostic work-up, as illustrated in a simple nutritional risk diagram (fig. 1) [16].

Dual energy X-ray absorptiometry (DEXA) is currently recommended for combined screening for osteoporosis, sarcopenia and adiposity as osteoporosis also appears to be a significant comorbidity and often coincides with muscle loss in COPD. Distinction between abdominal visceral and subcutaneous fat mass and muscular adiposity requires advanced and expensive imaging technologies (e.g. CT and magnetic resonance imaging). A clinically useful estimate of visceral fat may be derived by DEXA [27] but needs further validation. According to recent guidelines, sarcopenia is defined as appendicular lean mass index equal or below the mean minus 2 SD of that of healthy persons between 20 and 30 years of age from the same ethnic group [28]. In a representative adult population using DEXA data (13236 subjects aged $\geqslant 18$ years) obtained from the 1999 to 2004 National Health and Nutrition Examination Survey, PrADO et al. [29] developed sex- and BMI-specific decile groups of appendicular skeletal muscle index $\left(\mathrm{kg} \cdot \mathrm{m}^{-2}\right)$ and fat mass index $\left(\mathrm{kg} \cdot \mathrm{m}^{-2}\right)$. If these reference values better discriminate between clinically relevant metabolic phenotypes needs to be determined. 


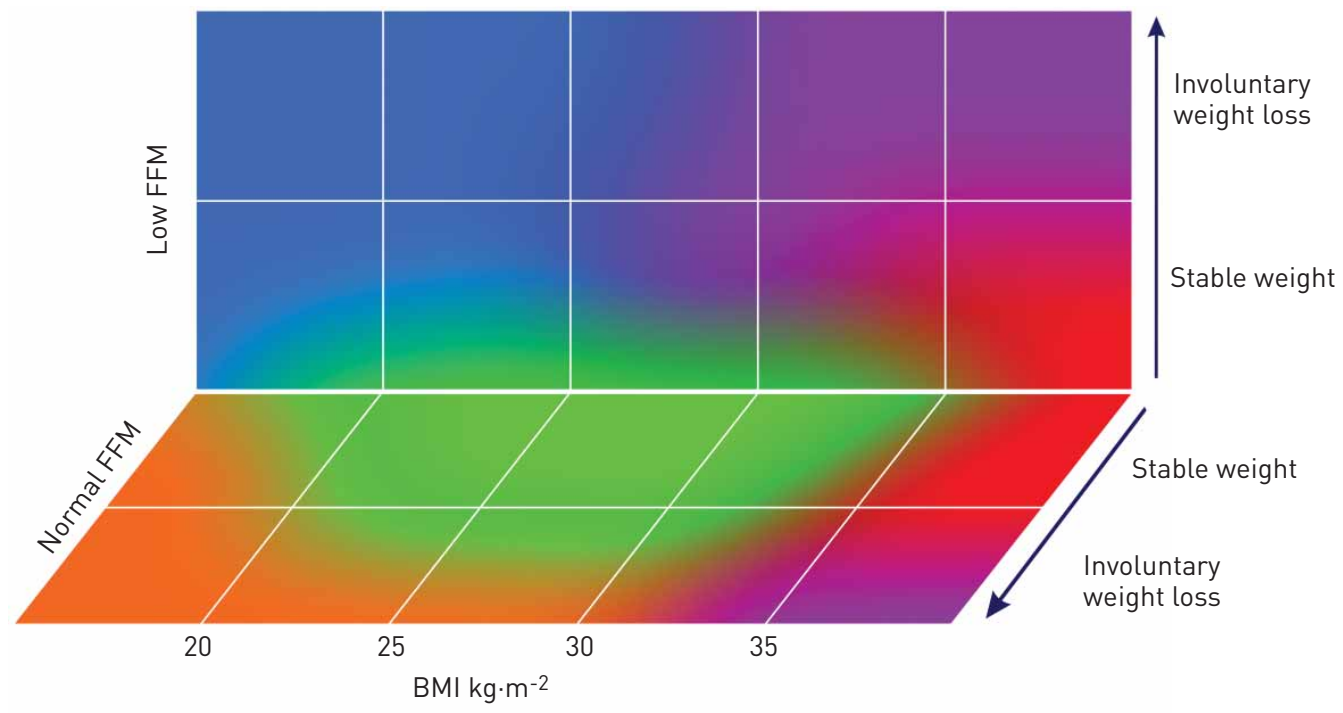

Low risk

Increased cardiovascular risk

Increased mortality risk

Decreased physical performance and increased mortality risk

Decreased physical performance and increased cardiovascular risk

FIGURE 1 Nutritional risk stratification diagram. FFM: fat free mass; BMI: body mass index. Reproduced from [16] with permission.

The advantages of using radiographic technology for the assessment of body composition is that this information can be collected at the time of routine imaging studies carried out for tumour evaluation and/ or restaging in lung cancer or for characterisation of emphysema in COPD. CT assessment of lumbar skeletal mass is a validated method to assess sarcopenia in (lung) cancer, which in turn is associated with increased mortality and risk for chemotherapy-induced toxicity [30]. Several studies in 2014 also evaluated the potential of chest CT-derived estimates of body composition in patients with COPD, including CT-assessed bone attenuation [31], muscle and fat infiltration of intercostal muscles [32] and CT measurements of the pectoralis muscle area [33]. In all three cross-sectional studies these estimates were related to measures of clinical outcome but, surprisingly, were not validated against references methods.

A disadvantage of most imaging procedures is the need for expensive equipment and the laboratory setting. Ultrasound has been investigated as a potential alternative but this method also requires more extensive validation. Several decades ago, 24-h urinary creatinine excretion was commonly used as biomarker of skeletal muscle mass which also appeared to be associated with exercise performance in COPD [34]. Recently, creatine (methyl-d3) dilution was presented as a new muscle mass biomarker [35]. The method is based on the principle that creatine (methyl-d3) dilution (D3-creatine) measured by enrichment of urine D3 creatinine reveals total body creatine pool size, providing an accurate estimate of total body skeletal muscle mass. The tracer is administered by a pill and the subjects only have to provide a urine sample at a standardised time-point. In a cross-sectional comparison, muscle or lean mass estimates by creatine dilution corresponded well with 24-h urinary creatinine excretion and appendicular muscle mass estimated by DEXA and magnetic resonance imaging. More research is needed to validate the method in detecting changes in muscle mass.

\section{Optimising anabolic potential in respiratory disease: a role for nutritional supplementation?}

PRADO et al. [36] conducted a quantitative analysis of CT images for the loss and gain of muscle in population-based cohorts of 368 advanced cancer patients, including lung cancer. Maintenance (45.6\%) or even increase in muscle mass (15.4\%) between two measurements indicated a window of opportunity for anabolic interventions in cancer at defined early phases of the disease trajectory. However, this approach also revealed that cancer patients within 90 days of death had a low likelihood of anabolic potential. Nutritional intervention is currently only considered in end-stage disease which may explain poor efficacy. An expert group, under the auspices of the European School of Oncology, recommend shifting therapeutic focus from end-stage wasting to supporting patients' nutritional and functional state throughout the increasingly complex and prolonged course of anti-cancer treatment [37]. 
The anabolic potential of nutritional intervention depends on the extraction of dietary nutrients, especially amino acids, by the intestine. RUTTEN et al. [38] provided evidence for disturbed intestinal integrity in patients with COPD, as measured by an increase of plasma intestinal fatty acid binding protein during activities of daily living and a decreased intestinal permeability. The clinical implications of this observation need to be determined. However, altered intestinal integrity does not seem to adversely affect the anabolic response to a meal in COPD. In contrast, JONKER et al. [39] and ENGELEN et al. [40] reported that a decreased splanchnic extraction of amino acids was associated with an enhanced anabolic response to a protein rich meal in sarcopenic COPD patients. In 2013 these results were extended to nutritionally depleted patients with COPD [39]. The latest meta-analyses have consistently shown that nutritional supplementation is able to improve body weight, fat-free mass and physical performance of nutritionally depleted patients with COPD [41-43]. The potential of nutritional intervention to augment exercise training responses is less convincing and may depend not only on nutritional status or COPD phenotype but also on the type and intensity of exercise training [44].

Nutrients, in particular amino acids, are able to stimulate muscle anabolism but the net effect on muscle mass depends on the degree of muscle protein breakdown (catabolism). No human data are available regarding muscle protein degradation rate in respiratory diseases due to methodological limitations, but some studies investigated muscle protein degradation signalling. Experimental studies have shown involvement of the ubiquitin proteasome pathway and the autophagy-lysosome pathway in disease-induced catabolism. Remarkably, there is only limited and inconsistent evidence for activation of the ubiquitin proteasome pathway in COPD [45] and, so far, no evidence in lung cancer. Conversely, in both conditions, increased autophagy was recently reported as a new indication, being most pronounced in patients with muscle wasting [46, 47]. Muscle maintenance, however, not only depends on muscle protein balance but also on the balance between myogenesis and apoptosis. Recently, in COPD [48] and cancer [49] evidence for impaired myogenesis was demonstrated, indicating that the regenerative capacity of muscle may be an additional target to combat muscle wasting in respiratory disease.

\section{Conclusion}

Analysis of recent literature shows that nutrition and metabolism are a hot topic in respiratory diseases. The clinical relevance of obesity was highlighted in primary and secondary prevention across all respiratory diseases. Radiographic imaging was explored for characterisation of metabolic phenotypes as an integrated part of the diagnostic work-up. Muscle regenerative defects and enhanced autography were identified as new leads for combatting muscle wasting in advanced disease.

\section{References}

1 Chung $\mathrm{JH}$, Hwang $\mathrm{HJ}$, Han $\mathrm{CH}$, et al. Association between sarcopenia and metabolic syndrome in chronic obstructive pulmonary disease: the Korea National Health and Nutrition Examination Survey (KNHANES) from 2008 to 2011. COPD 2014 [in press DOI: 10.3109/15412555.2014.908835].

2 Prado CM, Lieffers JR, McCargar LJ, et al. Prevalence and clinical implications of sarcopenic obesity in patients with solid tumours of the respiratory and gastrointestinal tracts: a population-based study. Lancet Oncol 2008; 9: 629-635.

3 Granell R, Henderson AJ, Evans DM, et al. Effects of BMI, fat mass, and lean mass on asthma in childhood: a Mendelian randomization study. PLoS Med 2014; 11: e1001669.

4 Yiallouros PK, Lamnisos D, Kolokotroni $\mathrm{O}$, et al. Associations of body fat percent and body mass index with childhood asthma by age and gender. Obesity 2013; 21: E474-E482.

5 Chen YC, Tu YK, Huang KC, et al. Pathway from central obesity to childhood asthma. Physical fitness and sedentary time are leading factors. Am J Respir Crit Care Med 2014; 189: 1194-1203.

6 Han YY, Forno E, Celedon JC. Adiposity, fractional exhaled nitric oxide, and asthma in U.S. children. Am J Respir Crit Care Med 2014; 190: 32-39.

7 Bradley GM, Blackman SM, Watson CP, et al. Genetic modifiers of nutritional status in cystic fibrosis. Am J Clin Nutr 2012; 96: 1299-1308.

8 Stephenson AL, Mannik LA, Walsh S, et al. Longitudinal trends in nutritional status and the relation between lung function and BMI in cystic fibrosis: a population-based cohort study. Am J Clin Nutr 2013; 97: 872-877.

9 Panagopoulou P, Fotoulaki M, Nikolaou A, et al. Prevalence of malnutrition and obesity among cystic fibrosis patients. Pediatr Int 2014; 56: 89-94.

10 Hanna RM, Weiner DJ. Overweight and obesity in patients with cystic fibrosis: a center-based analysis. Pediatr Pulmonol 2015; 50: 35-41.

11 Behrens G, Matthews CE, Moore SC, et al. Body size and physical activity in relation to incidence of chronic obstructive pulmonary disease. CMAJ 2014; 186: E457-E469.

12 Varraso R, Fung TT, Hu FB, et al. Prospective study of dietary patterns and chronic obstructive pulmonary disease among US men. Thorax 2007; 62: 786-791.

13 Varraso R, Jiang R, Barr RG, et al. Prospective study of cured meats consumption and risk of chronic obstructive pulmonary disease in men. Am J Epidemiol 2007; 166: 1438-1445.

14 Fonseca Wald EL, van den Borst B, Gosker HR, et al. Dietary fibre and fatty acids in chronic obstructive pulmonary disease risk and progression: a systematic review. Respirology 2013; 19: 176-184.

15 Vanfleteren LE, Spruit MA, Groenen M, et al. Clusters of comorbidities based on validated objective measurements and systemic inflammation in patients with chronic obstructive pulmonary disease. Am J Respir Crit Care Med 2013; 187: 728-735. 
16 Schols AM, Ferreira IM, Franssen FM, et al. Nutritional assessment and therapy in COPD: a European Respiratory Society statement. Eur Respir J 2014; 44: 1504-1520.

17 Van Remoortel H, Hornikx M, Langer D, et al. Risk factors and comorbidities in the preclinical stages of chronic obstructive pulmonary disease. Am J Respir Crit Care Med 2014; 189: 30-38.

18 van den Borst B, Koster A, Yu B, et al. Is age-related decline in lean mass and physical function accelerated by obstructive lung disease or smoking? Thorax 2011; 66: 961-969.

19 van den Borst B, Gosker HR, Koster A, et al. The influence of abdominal visceral fat on inflammatory pathways and mortality risk in obstructive lung disease. Am J Clin Nutr 2012; 96: 516-526.

20 van den Borst B, Gosker HR, Schols AM. Central fat and peripheral muscle: partners in crime in chronic obstructive pulmonary disease. Am J Respir Crit Care Med 2013; 187: 8-13.

21 van den Borst B, Slot IG, Hellwig VA, et al. Loss of quadriceps muscle oxidative phenotype and decreased endurance in patients with mild-to-moderate COPD. J Appl Physiol (1985) 2013; 114: 1319-1328.

22 Gagnon P, Lemire BB, Dube A, et al. Preserved function and reduced angiogenesis potential of the quadriceps in patients with mild COPD. Respir Res 2014; 15: 4.

23 Remels AH, Gosker HR, Langen RC, et al. Classical NF-kappaB activation impairs skeletal muscle oxidative phenotype by reducing IKK-alpha expression. Biochim Biophys Acta 2014; 1842: 175-185.

24 Natanek SA, Gosker HR, Slot IG, et al. Heterogeneity of quadriceps muscle phenotype in chronic obstructive pulmonary disease (Copd); implications for stratified medicine? Muscle Nerve 2013; 48: 488-497.

25 Gouzi F, Abdellaoui A, Molinari N, et al. Fiber atrophy, oxidative stress, and oxidative fiber reduction are the attributes of different phenotypes in chronic obstructive pulmonary disease patients. J Appl Physiol (1985) 2013; 115: 1796-1805.

26 Maddocks M, Shrikrishna D, Vitoriano S, et al. Skeletal muscle adiposity is associated with physical activity, exercise capacity and fibre shift in COPD. Eur Respir J 2014; 44: 1188-1198.

27 Micklesfield LK, Goedecke JH, Punyanitya M, et al. Dual-energy X-ray performs as well as clinical computed tomography for the measurement of visceral fat. Obesity (Silver Spring) 2012; 20: 1109-1114.

28 Cruz-Jentoft AJ, Baeyens JP, Bauer JM, et al. Sarcopenia: European consensus on definition and diagnosis: Report of the European Working Group on Sarcopenia in older people. Age Ageing 2010; 39: 412-423.

29 Prado CM, Siervo M, Mire E, et al. A population-based approach to define body-composition phenotypes. Am J Clin Nutr 2014; 99: 1369-1377.

30 Martin L, Birdsell L, Macdonald N, et al. Cancer cachexia in the age of obesity: skeletal muscle depletion is a powerful prognostic factor, independent of body mass index. J Clin Oncol 2013; 31: 1539-1547.

31 Romme EA, Murchison JT, Edwards LD, et al. CT-measured bone attenuation in patients with chronic obstructive pulmonary disease: relation to clinical features and outcomes. J Bone Miner Res 2013; 28: 1369-1377.

32 Park MJ, Cho JM, Jeon KN, et al. Mass and fat infiltration of intercostal muscles measured by CT histogram analysis and their correlations with COPD severity. Acad Radiol 2014; 21: 711-717.

33 McDonald ML, Diaz AA, Ross JC, et al. Quantitative computed tomography measures of pectoralis muscle area and disease severity in chronic obstructive pulmonary disease. A cross-sectional study. Ann Am Thorac Soc 2014; 11: $326-334$.

34 Schols AM, Mostert R, Soeters PB, et al. Body composition and exercise performance in patients with chronic obstructive pulmonary disease. Thorax 1991; 46: 695-699.

35 Clark RV, Walker AC, O'Connor-Semmes RL, et al. Total body skeletal muscle mass: estimation by creatine (methyl-d3) dilution in humans. J Appl Physiol (1985) 2014; 116: 1605-1613.

36 Prado CM, Sawyer MB, Ghosh S, et al. Central tenet of cancer cachexia therapy: do patients with advanced cancer have exploitable anabolic potential? Am J Clin Nutr 2013; 98: 1012-1019.

37 Aapro M, Arends J, Bozzetti F, et al. Early recognition of malnutrition and cachexia in the cancer patient: a position paper of a European School of Oncology Task Force. Ann Oncol 2014; 25: 1492-1499.

38 Rutten EP, Lenaerts K, Buurman WA, et al. Disturbed intestinal integrity in patients with COPD: effects of activities of daily living. Chest 2014; 145: 245-252.

39 Jonker R, Deutz NE, Erbland ML, et al. Hydrolyzed casein and whey protein meals comparably stimulate net whole-body protein synthesis in COPD patients with nutritional depletion without an additional effect of leucine co-ingestion. Clin Nutr 2014; 33: 211-220.

40 Engelen MP, De Castro CL, Rutten EP, et al. Enhanced anabolic response to milk protein sip feeding in elderly subjects with COPD is associated with a reduced splanchnic extraction of multiple amino acids. Clin Nutr 2012; 31: 616-624.

41 Ferreira IM, Brooks D, White J, et al. Nutritional supplementation for stable chronic obstructive pulmonary disease. Cochrane Database Syst Rev 2012; 12: CD000998.

42 Collins PF, Stratton RJ, Elia M. Nutritional support in chronic obstructive pulmonary disease: a systematic review and meta-analysis. Am J Clin Nutr 2012; 95: 1385-1395.

43 Collins PF, Elia M, Stratton RJ. Nutritional support and functional capacity in chronic obstructive pulmonary disease: a systematic review and meta-analysis. Respirology 2013; 18: 616-629.

44 Constantin D, Menon MK, Houchen-Wolloff L, et al. Skeletal muscle molecular responses to resistance training and dietary supplementation in COPD. Thorax 2013; 68: 625-633.

45 Langen RC, Gosker HR, Remels $\mathrm{AH}$, et al. Triggers and mechanisms of skeletal muscle wasting in chronic obstructive pulmonary disease. Int I Biochem Cell Biol 2013; 45: 2245-2256.

46 Guo Y, Gosker HR, Schols AM, et al. Autophagy in locomotor muscles of patients with chronic obstructive pulmonary disease. Am J Respir Crit Care Med 2013; 188: 1313-1320.

47 Op den Kamp CM, Langen RC, Snepvangers FJ, et al. Nuclear transcription factor kappa B activation and protein turnover adaptations in skeletal muscle of patients with progressive stages of lung cancer cachexia. Am J Clin Nutr 2013; 98: 738-748.

48 Theriault ME, Pare ME, Lemire BB, et al. Regenerative defect in vastus lateralis muscle of patients with chronic obstructive pulmonary disease. Respir Res 2014; 15: 35.

49 He WA, Berardi E, Cardillo VM, et al. NF-kappaB-mediated Pax7 dysregulation in the muscle microenvironment promotes cancer cachexia. J Clin Invest 2013; 123: 4821-4235. 